\section{Alas1 is essential for neutrophil maturation in zebrafish}

\author{
Junwei Lian, ${ }^{1}$ Jiakui Chen, ${ }^{1}$ Kun Wang, ${ }^{1}$ Lingfeng Zhao, ${ }^{1}$ Ping Meng, ${ }^{1}$ \\ Liting Yang, ${ }^{1}$ Jiayi Wei, ${ }^{1}$ Ning Ma, ${ }^{1}$ Jin $\mathrm{Xu}^{2}{ }^{2}$ Wenqing Zhang ${ }^{2}$ and Yiyue Zhang ${ }^{1,2}$ \\ ${ }^{1}$ Key Laboratory of Zebrafish Modeling and Drug Screening for Human Diseases of \\ Guangdong Higher Education Institutes, Department of Developmental Biology, School \\ of Basic Medical Sciences, Southern Medical University and 'Division of Cell, \\ Developmental and Integrative Biology, School of Medicine, South China University of \\ Technology, Guangzhou, P.R. China
}

\section{ABSTRACT}

$\mathrm{N}$ eutrophils play essential roles in innate immunity and are the first responders to kill foreign micro-organisms, a function that partially depends on their granule content. The complicated regulatory network of neutrophil development and maturation remains largely unknown. Here we utilized neutrophil-deficient zebrafish to identify a novel role of Alas1, a heme biosynthesis pathway enzyme, in neutrophil development. We showed that Alas1-deficient zebrafish exhibited proper neutrophil initiation, but further neutrophil maturation was blocked due to heme deficiency, with lipid storage and granule formation deficiencies, and loss of heme-dependent granule protein activities. Consequently, Alas1-deficient zebrafish showed impaired bactericidal ability and augmented inflammatory responses when challenged with Escherichia coli. These findings demonstrate the important role of Alas1 in regulating neutrophil maturation and physiological function through the heme. Our study provides an in vivo model of Alas1 deficiency and may be useful to evaluate the progression of heme-related disorders in order to facilitate the development of drugs and treatment strategies for these diseases.

\section{Introduction}

Neutrophils are the most abundant leukocytes in the circulation and the first responders to sites of infection, where they attack pathogens by phagocytosis, degranulation, and by generating neutrophil extracellular traps. ${ }^{1,2}$ Neutrophil development is highly conserved in vertebrates, making zebrafish a suitable model for investigation. Neutrophils are derived from granulocyte-monocyte progenitors, and undergo determination and differentiation from myeloblasts to mature neutrophils. ${ }^{1,3}$ During neutrophil differentiation and maturation, neutrophil granules are formed and assembled. ${ }^{1}$ Anti-microbial proteins are thought to be the major constituents of neutrophil granules, and they play important roles in neutrophil diapedesis, chemotaxis, and the phagocytosis of micro-organisms. ${ }^{1,4}$

Several transcription factors have been reported to be involved in neutrophil development and physiological function in mammals, including SPI.1/PU1 and C/EBP-E. ${ }^{3}$ A recent study of embryonic myelopoiesis revealed that the Pu.1-Runx1 regulatory loop controlled embryonic myeloid cell fate in zebrafish. ${ }^{5,6} \mathrm{We}$ previously demonstrated that c-Myb and Cebp1 co-operatively acted in parallel to govern neutrophil maturation. ${ }^{7}$ In addition to these transcription factors, zebrafish deficient for the neutrophil granule protein myeloperoxidase (Mpx) or the neutrophilspecific marker nephrosin (Npsn) have altered neutrophil maturation and inflammatory responses to fungal and bacterial infection, respectively., Nevertheless, the complicated regulatory network of neutrophil maturation, as well as the impact on physiological function remain poorly understood.

Heme (iron protoporphyrin IX) functions as a prosthetic group on various proteins, so-called hemoproteins, such as hemoglobin, myoglobin, cytochromes, catalases, and peroxidases. ${ }^{10}$ Hemoproteins are involved in diverse biological functions, including oxygen transport, energy metabolism, and drug biotransformation. ${ }^{11}$ Moreover, heme also plays important roles in the regulation of transcription, ${ }^{12-14}$

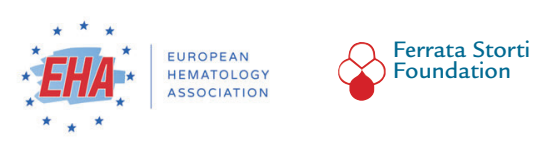

Haematologica 2018

Volume 103(11):1785-1795

\section{Correspondence:}

yiyue@smu.edu.cn or mczhangyy@scut.edu.cn or mczhangwq@scut.edu.cn

Received: March 27, 2018.

Accepted: June 27, 2018.

Pre-published: June 28, 2018

doi:10.3324/haematol.2018.194316

Check the online version for the most updated information on this article, online supplements, and information on authorship \& disclosures: www.haematologica.org/content/103/11/1785

(C)2018 Ferrata Storti Foundation

Material published in Haematologica is covered by copyright. All rights are reserved to the Ferrata Storti Foundation. Use of published material is allowed under the following terms and conditions:

https://creativecommons.org/licenses/by-nc/4.0/legalcode. Copies of published material are allowed for personal or internal use. Sharing published material for non-commercial purposes is subject to the following conditions:

https://creativecommons.org/licenses/by-nc/4.0/legalcode, sect. 3. Reproducing and sharing published material for commercial purposes is not allowed without permission in writing from the publisher. 
translation, ${ }^{15,16}$ protein degradation, ${ }^{17,18}$ and microRNA processing. ${ }^{19}$ The accumulation of excess heme and its precursors in tissues can cause oxidative damage via the generation of reactive oxygen species. ${ }^{11}$ Thus, cellular heme homeostasis must be tightly controlled.

The 5 -aminolevulinate synthase 1 (ALAS1) is a mitochondrial enzyme that catalyzes the condensation of glycine and succinyl-CoA, forming 5-aminolevulinic acid. ALAS1 is the first and rate-limiting enzyme of the heme biosynthetic pathway, which is conserved from lower to higher organisms. ${ }^{20}$ ALAS1 (also called hepatic ALAS or non-specific ALAS) is ubiquitously expressed throughout the body, whereas another isoform, ALAS2 (also called ALAS-E), is predominantly expressed in erythroid cells, to meet the need of the large amounts of heme required for hemoglobin synthesis. ${ }^{11}$ It has been reported that human ALAS2 mutations cause X-linked sideroblastic anemia; ${ }^{21}$ ALAS2-deficient mice and zebrafish also display severe anemia, ${ }^{22,23}$ revealing the major contribution of ALAS2 to erythroid heme biosynthesis, and hence how it is essential to erythroid differentiation. In contrast to ALAS2, there are no reported human diseases directly caused by mutations in ALAS1. In mice, Alas1-null embryos are lethal by embryonic day 8.5 (E8.5), ${ }^{24}$ thus, the in vivo physiological role of ALAS1 is unclear. Using GFP knock-in mice $\left(\right.$ Alas $\left.1^{+/ G F P}\right)$, ALAS1 was found to be highly expressed in the liver, exocrine, endocrine glands, and myeloid cells, where large amounts of heme are required to meet the needs of tissue-specific hemoproteins, such as MPO, NADPH oxidase, and CYP450. ${ }^{24}$ Notably, Alas 1 is also expressed higher in neutrophils than macrophages,$^{24}$ suggesting cell-specific roles in neutrophils. However, the function of ALAS1 in neutrophils is still unknown.

Taking advantage of their transparent body, we can observe neutrophil morphology to trace neutrophil behaviors in live zebrafish. Zebrafish is an ideal model for studying neutrophil biology. ${ }^{25,26}$ Here we report a novel role of Alas1 in regulating neutrophil development using a neutrophil lineage-deficient mutant zebrafish line (previously named smu350) that was isolated from our ENU mutant collection. ${ }^{27}$ We demonstrated that alas 1 was the causative gene for the mutant and revealed that alas 1 was essential for neutrophil development and physiological function.

\section{Methods}

\section{Fish maintenance}

Zebrafish were raised and maintained under standard conditions. ${ }^{28}$ Embryos were maintained in egg water containing $0.2 \mathrm{mM}$ $\mathrm{N}$-phenylthiourea (Sigma-Aldrich, St. Louis, MO, USA) to prevent pigment formation. All work involving zebrafish was approved by Southern Medical University Animal Ethics Committee. The following strains were used: $\mathrm{AB}, \mathrm{Tg}(\mathrm{lyz}: \mathrm{Ds} R e d){ }^{29} \mathrm{Tg}$ (gata1:DsRed),

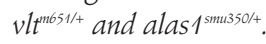

\section{Treatment with succinylacetone}

Succinylacetone (SA) (Sigma-Aldrich, St. Louis, MO, USA) was dissolved in egg water. Zebrafish embryos were placed in culture dishes containing $1 \mathrm{mM} \mathrm{SA}$ at 5 hours (h) post fertilization (hpf) till the desired stage.

\section{Bacterial infection}

The eGFP-labeled E. coli strain XL10 was cultured in LB broth with $50 \mu \mathrm{g} \mathrm{mL}-1$ ampicillin at $37^{\circ} \mathrm{C}$ until reaching an optical den- sity at $600 \mathrm{~nm}$ between $0.5-0.8$. Bacteria were washed with sterile phosphate buffered saline (PBS) three times, harvested by centrifugation at $5000 \mathrm{x}$ g for 5 minutes (min) and resuspended in sterile PBS. The working concentration of E. coli was $2 \times 10^{9} \mathrm{~mL}-1$, and approximately $0.5 \mathrm{~nL}$ of bacterial suspension was subcutaneously injected over a somite into 3-day-post-fertilization (dpf) embryos with $0.02 \%$ tricaine using a PLI-100A Pico-Injector (Warner Instruments, Hamden, CT, USA) as previously described. ${ }^{26,30}$ For bacterial colony forming assays, every injected embryo was washed with sterile PBS three times, and then homogenized in $200 \mu \mathrm{L}$ of sterile PBS at the desired time points. Then, $10 \mu \mathrm{L}$ of homogenate was plated on LB medium with ampicillin and cultured at $37^{\circ} \mathrm{C}$ overnight. The results are the average of two separate experiments.

\section{Statistical analysis}

Data were recorded and analyzed using GraphPad Prism 7 and IBM SPSS v.23. Two-tailed Student $t$-test and Mann-Whitney U test were used for comparisons between parametric and non-parametric data, respectively. One-way analysis of variance (with Bonferroni or Dunnett T3 post-test adjustment) was used for parametric data to make multi-comparisons. Differences were considered significant at $P<0.05$. Data are expressed as the mean \pm Standard Deviation (SD).

\section{Results}

\section{Neutrophil deficiency in smu350 mutant zebrafish}

To identify new regulators of neutrophil development, we conducted a genetic screen for neutrophil-deficient zebrafish mutants using Sudan black B (SB) staining. From this screen, we isolated the neutrophil-deficient smu 350 mutant, which lacked the SB signal as early as $36 \mathrm{hpf}$ (Figure 1A). The early loss of the SB signal in smu350 mutants suggested defects in embryonic neutrophils, as $\mathrm{SB}^{+}$cells represent embryonic neutrophils that are initiating from embryonic myelopoietic tissue. ${ }^{31}$ Because myeloid progenitors that are derived from rostral blood islands will progress to neutrophils during embryonic myelopoiesis, we first determined if there were defects in the formation of myeloid progenitors. The results showed that pu. 1 expression at $22 \mathrm{hpf}$ was normal (Figure 1B), suggesting the presence of myeloid progenitors in smu 350 mutants. Therefore, we speculated that the loss of the SB signal was due to defects in neutrophil maturation. To test this possibility, we detected the transcript and protein activity of the myeloid-specific peroxidase $(M p x)$, which is an abundant granule protein in neutrophils. ${ }^{2}$ Wholemount in situ hybridization (WISH) showed that $m p x$ mRNA expression was intact (Figure 1C), suggesting the presence of neutrophils in smu350 mutants. To further examine Mpx enzyme activity, diaminobenzidine (DAB, a peroxidase substrate) staining was performed. The results showed that while the signal in the yolk sac (representing hemoglobin peroxidase activity ${ }^{32}$ ) was present, signals representing neutrophil peroxidase activity were absent in smu350 mutants (Figure 1D), suggesting that Mpx lost its catalytic activity. As neutrophil granules are abundant with Mpx, we directly monitored neutrophil granule morphology via video-enhanced differential interference contrast (VE-DIC) analyses of live embryos. The results showed that neutrophils from siblings (alas $1^{\text {smmun } 300 /+}$ and alas $1^{1+4}$ embryos from a heterozygous alas $1^{\text {smum } 300 /+}$ in-cross) had abundant visible and highly mobilized granules, while neutrophils from smu350 mutants lacked such granules 
(Figure 1E and Online Supplementary Appendix, Movies 1 and 2). These results suggest that neutrophil maturation is defective in smu350 mutants.

\section{The alas1 mutation was responsible for neutrophil defects of smu350 mutant}

Positional cloning was then performed to identify the causative gene in smu350 mutants. Initial mapping with bulk segregation analysis located the mutated site to link- age group 11 (data not shown), then fine mapping placed the mutated gene within a region between two simple sequence length polymorphism markers, CU633745-M and CU929297-M, from the Massachusetts General Hospital panel (Figure 2A). The mutation was then mapped to a $100-\mathrm{kb}$ region partly covered by two bacterial artificial chromosomes (BACs) (Figure 2A). There were 9 predicted genes in this region (Figure 2A). By sequencing, we found a T-to-A mutation in alas 1 intron 7 next to the

Sibling

Smu350 mutant

A

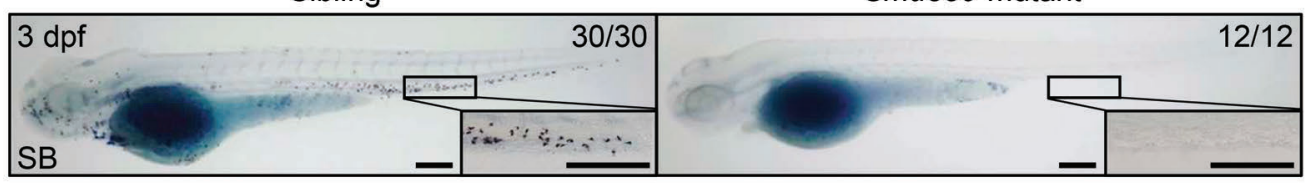

B

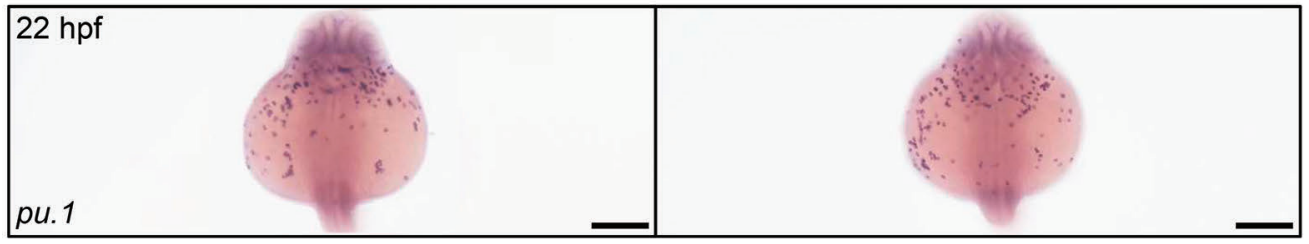

C

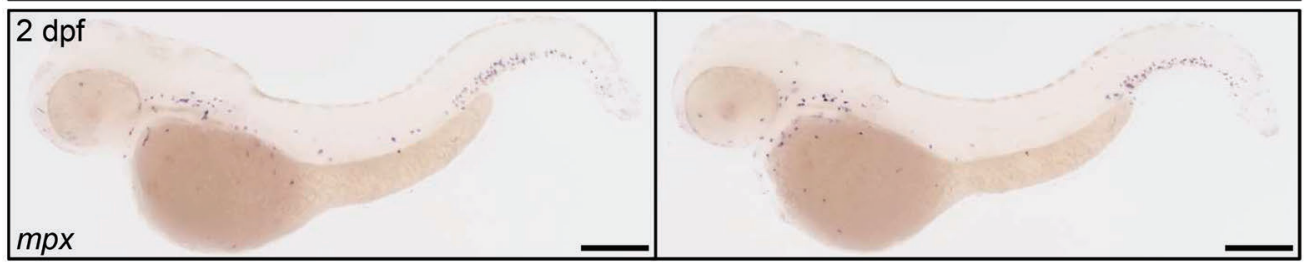

D

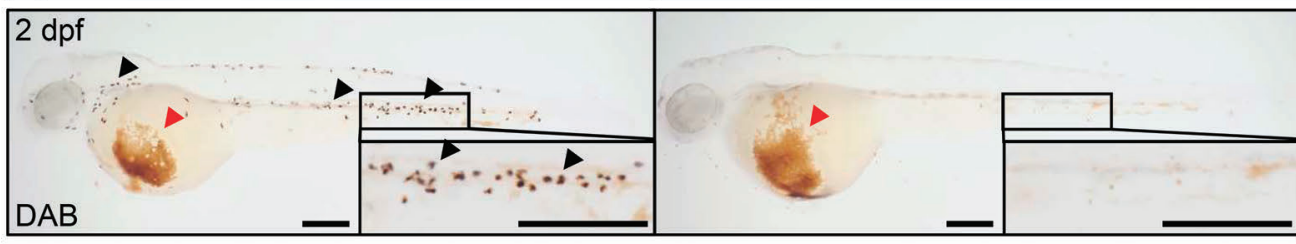

$E$

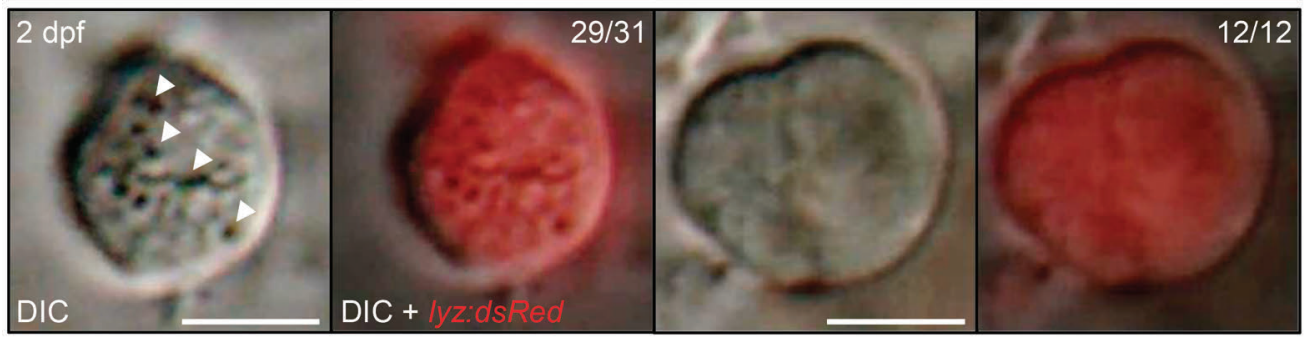

$\mathbf{F}$

Sibling

Smu350

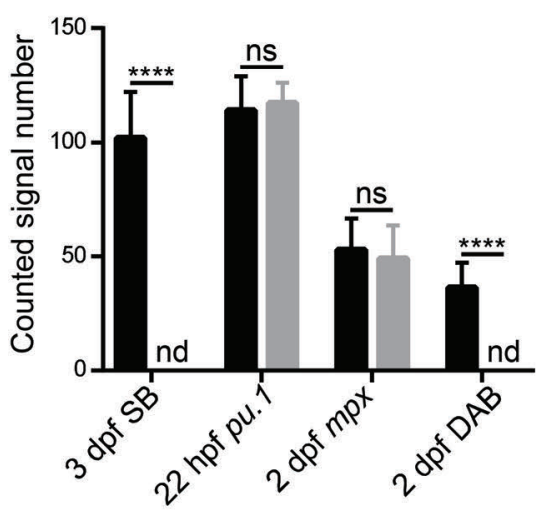

Figure 1. Neutrophil deficiency in smu350 mutants. (A) The Sudan black B (SB) signal was totally absent in smu350 mutants. SB staining in siblings (left) and smu350 mutants (right) at 3 days post fertilization (dpf). The boxed regions are magnified in the lower right-hand corner. (B) Whole-mount in situ hybridization (WISH) of pu.1 expression in siblings (left) and smu350 mutants (right) at 22 hours post fertilization (hpf). (C) WISH of mpx expression in siblings (left) and smu350 mutants (right) at $2 \mathrm{dpf}$. (D) DAB staining in siblings (left) and smu350 mutants (right) at $2 \mathrm{dpf}$. Signal points representing neutrophil peroxidase activity (black arrowheads) were absent in smu350 mutants. Red arrowheads indicate the hemoglobin signal. The boxed regions are magnified in the lower right-hand corner. (E) Neutrophil granules were absent in smu350 mutants. In vivo imaging of neutrophils in 2-dpf $\mathrm{Tg}(\mathrm{lyz}$ :DsRed);smu350 embryos by VE-DIC microscopy. Neutrophils of siblings had abundant visible and highly mobilized granules (29 of 31 embryos), while neutrophils of smu350 mutants lacked granules (12 of 12 embryos). White arrowheads indicate neutrophil granules. (Left) Bright-field DIC image; (right) an overlay of bright-field DIC and fluorescent images. See also Online Supplementary Appendix, Movies 1 and 2. (F) Quantifications of $\mathrm{SB}^{+}$cells in the posterior blood island (PBI), pu. $1^{+}$cells $(\mathrm{B}), m^{+}$cells in the $\mathrm{PBI}(\mathrm{C})$, and $\mathrm{DAB}^{+}$cells in the PBI (D). Mean \pm Standard Deviation (SD), $n>15$; Student $t$-test: $* * * * P<0.0001$. ns: not significant; nd: not detectable. Scale bars: $200 \mu \mathrm{m}$ (A-D) and $5 \mu \mathrm{m}$ (E). 
A

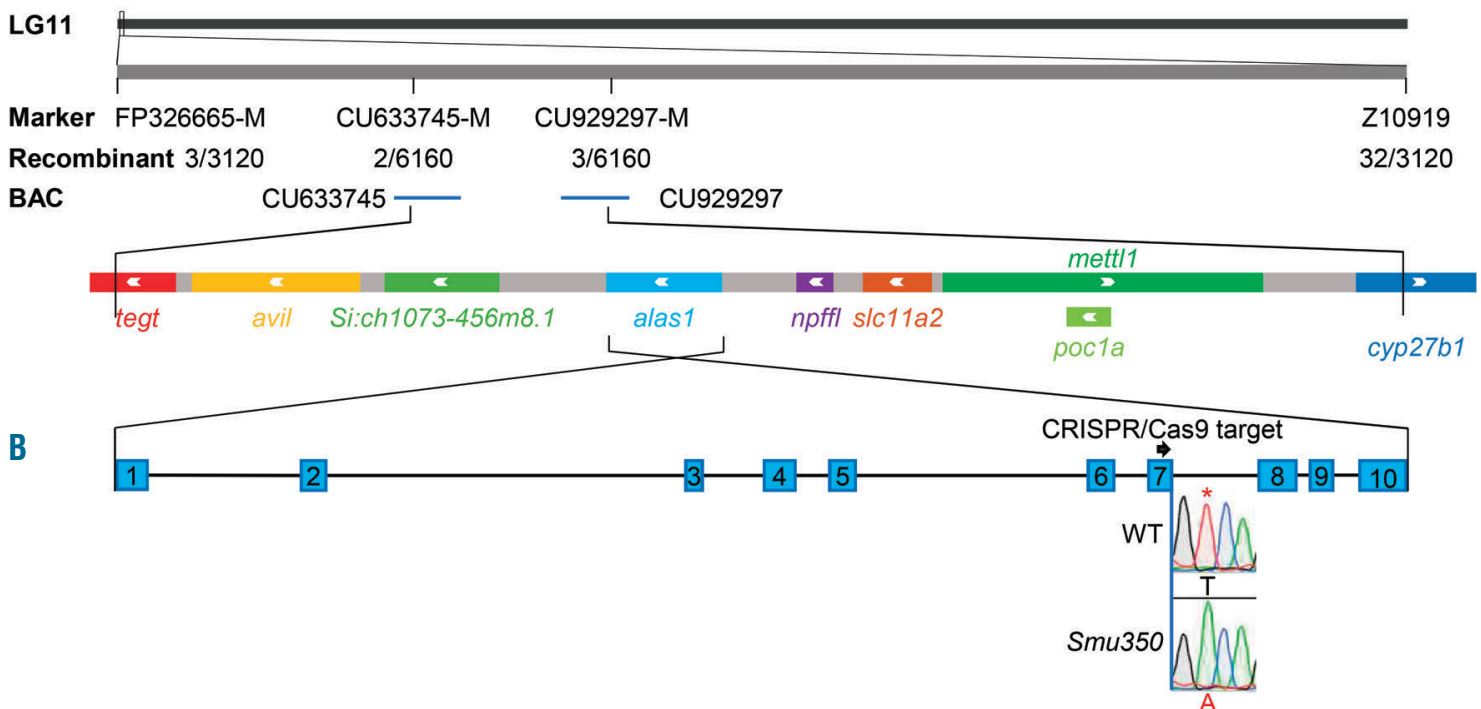

C

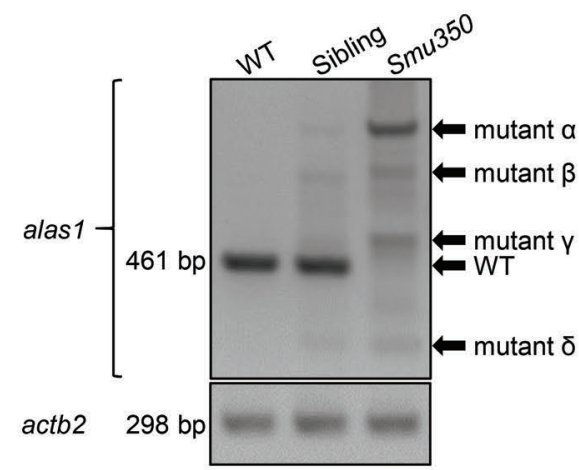

D

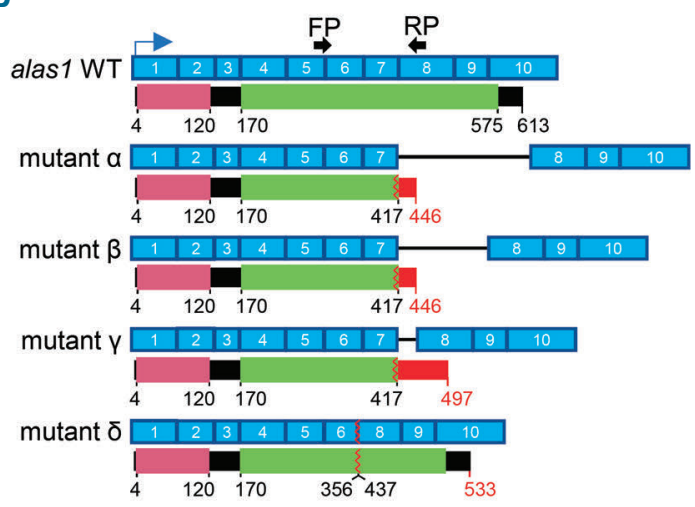

$\mathrm{E}$

WT Smu350

F
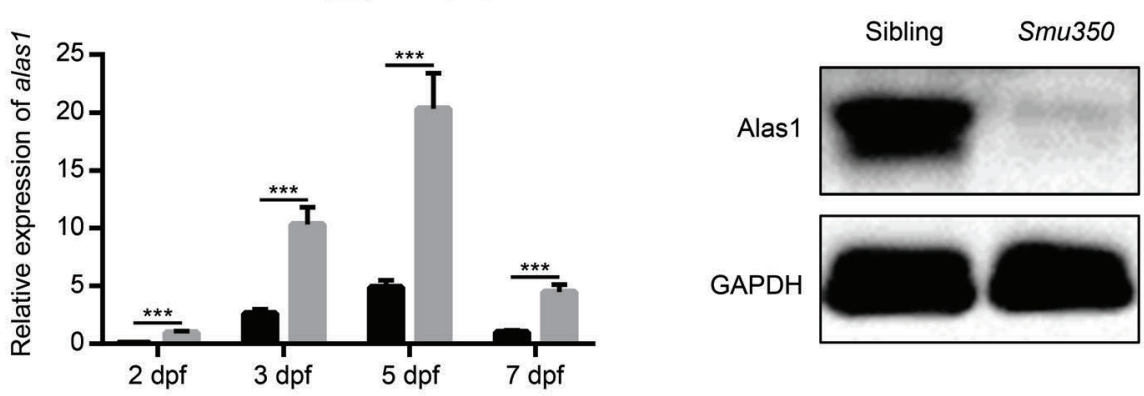

Figure 2. The alas1 gene was mutated in smu350 mutants. (A) The mutated gene in smu350 mutants mapped to a 100-kb region between two simple sequence length polymorphism markers, CU633745-M (two recombinants in 6160 smu350 mutant embryos) and CU929297-M (three recombinants in 6160 smu350 mutant embryos), on linkage group 11. The 100-kb region, partly covered by two bacterial artificial chromosomes (BACs) (CU633745 and CU929297), contains 9 predicted genes. (B) The structure of the zebrafish alas1 gene. The red asterisk indicates a T-to-A mutation in intron 7 of alas 1 in smu350 mutants. The black arrow indicates the position of the CRISPR/Cas9 target in alas1. Numbers of constitutive exons are indicated. (C) Agarose gel electrophoresis of alas1 RT-qPCR amplification products from 3-day post fertilization (dpf) wild-type zebrafish, siblings, and smu350 mutants. Four major products (indicated by black arrows) were identified in smu350 mutants compared with wild-type transcripts (461 bp). The actb2 was used as an internal control. (D) The mutated alas1 transcripts and their predicted translation products in smu350 mutants. The blue arrow indicates the position of the transcriptional start site. Black arrows indicate the RT-qPCR primers used in (C), and black boxes indicate the wild-type peptides. Red boxes indicate the incorrect peptides generated by the altered splicing. Pink boxes indicate the pre-sequence domain (pfam09029). Green boxes indicate the aspartate aminotransferase superfamily domain (fold type I) of pyridoxal phosphate-dependent enzymes (cl18945). Boxes with zigzag edges indicate truncated regions. Blue boxes with white numbers indicate exons. Black and red numbers denote distances to the start codon in wildtype and mutants, respectively. (E) alas1 expression was up-regulated in smu350 mutants. Relative expression of alas 1 transcript assessed by RT-qPCR in smu350 mutants (gray column) and wild-type (black column) at 2, 3, 5, and $7 \mathrm{dpf}$ [mean \pm Standard Deviation (SD); $n=10$ in each group, performed in triplicate]. Statistical significance was determined using Student $t$-test, $* * * P<0.001$. (F) Alas1 protein was absent in smu350 mutants. Alas 1 protein expression in the whole fish body assessed by western blotting at $5 \mathrm{dpf}$. GAPDH was used as the loading control. 
exon-intron boundary in smu350 mutants (Figure 2B), which is likely to be a splicing mutation. By amplifying alas1 cDNA from smu350 mutants, we found at least four unexpected alas 1 transcripts (Figure 2C), which were confirmed by sequencing analysis following TA cloning. These unexpected transcripts were predicted to produce truncated Alas1 proteins or in-frame-insertion Alas1 proteins, all of which would interrupt the enzyme activity domain of Alas1 (Figure 2D). Expression analyses by reverse transcription quantitative real-time polymerase chain reaction (RT-qPCR) showed elevated alas 1 mRNA expression in smu350 mutants compared with siblings throughout development (Figure 2E). However, we found that neither the wild-type form nor the abnormal variants of Alas1 protein were present in smu350 mutants, as determined by western blotting (Figure $2 \mathrm{~F}$ ). These data strongly suggest that this alas1 mutation is responsible for the smu350 mutant (hereafter named alas $1^{\text {smu } 350 / \text { smu } 350}$ ) phenotype and that the alas $1^{\text {smu } 350 / \text { smu } 350}$ mutant is a loss-of-function mutant.

To confirm that the alas $1^{\text {smu } 350 / \text { smu } 330}$ mutant phenotype was indeed caused by the alast mutation, we used CRISPR/Cas9 to create alas1-knock-out mutants. A homozygous alas 1 mutant (alas $1^{\Delta 2 / \Delta^{2}}$ ) with a 2-bp deletion within exon 7 of alas1 was obtained, and the mutation resulted in a frameshift of the alas1 product, causing a loss of Alas1 protein in the alas $1^{12 / \Delta 2}$ mutant (Figure $3 \mathrm{~A}$ and B). Similar to the alas $1^{1 \mathrm{smu} 350 / \mathrm{smu} u 350}$ mutant, the alas $1^{4^{2 / 12}}$ homozygous mutant and the alas $1^{\text {smu } 350 / 42}$ bi-allelic mutant also showed loss of SB staining (Figure $3 \mathrm{C}$ and D), indicating that alas 1 is indeed the causative gene for the altered neutrophil development phenotype.

\section{The alas1 mutation caused heme deficiency}

ALAS1 is the first and rate-limiting enzyme for heme biosynthesis, and heme negatively regulates ALAS1 expression through a feedback mechanism. ${ }^{10}$ As the Alas1 protein was undetectable (Figure $2 \mathrm{~F}$ ), we postulated that the heme levels of alas $1^{\text {smu } 350 / \text { smu } 350}$ mutants might be decreased. To test this hypothesis, we measured heme levels in alas $1^{\text {smu } 350 / \text { smu } 350}$ mutants using a fluorescence heme assay. Surprisingly, total heme levels of alas $1^{\text {smu } 350 / \mathrm{smu}_{3} 30}$ mutants were abnormally elevated (Figure 4A).

As erythroid tissue is the major site of heme production in the body and depends on the isozyme Alas $2,{ }^{33}$ we then checked if this elevated heme was derived from erythrocytes. We first compared the erythrocyte numbers between 4-dpf alas $1^{\text {smu } 350 / \text { smu } 350}$ mutants and their siblings and
A
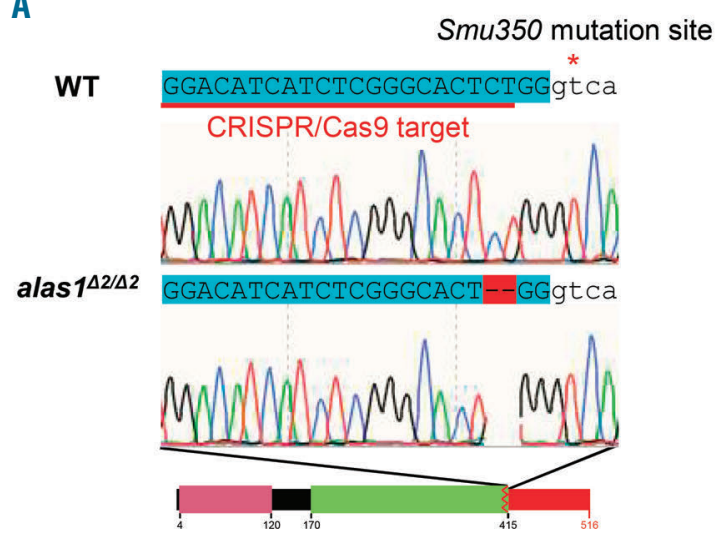

C

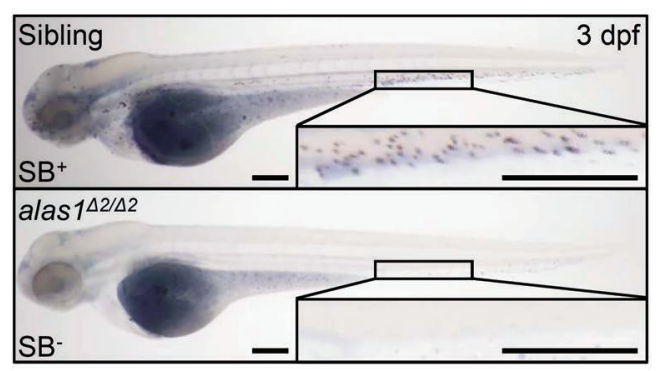

B

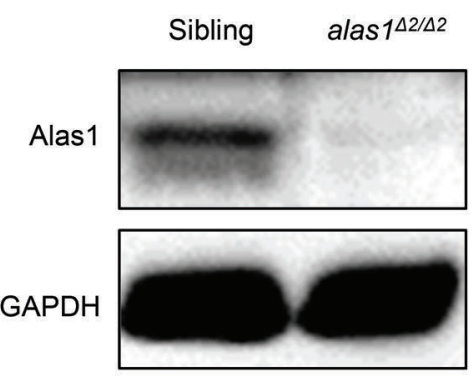

D

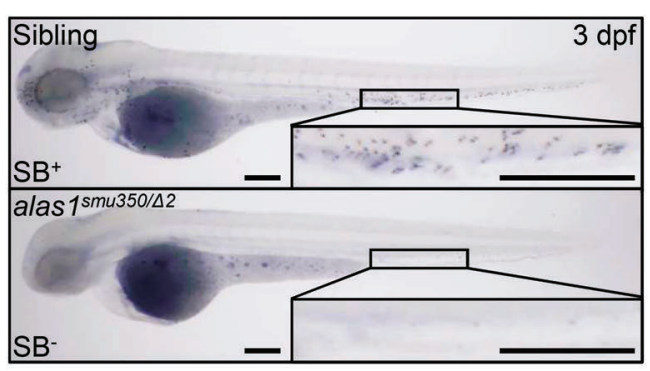

Figure 3. The alas 1 was the causative gene of the smu350 mutant. (A) Sequencing analysis revealed a 2-bp deletion within exon 7 of alas 1 in CRISPR/Cas 9 generated alas $1^{12 / 12}$ mutants. Uppercase sequences highlighted blue indicate exons; sequences in lowercase indicate introns. Sequences underlined in red indicate the CRISPR/Cas 9 target in alas1. The red asterisk indicates the smu350 mutation site. Black boxes indicate the wild-type peptides, and the red box indicates the incorrect peptide generated by the altered splicing. The pink box indicates the pre-sequence domain (pfam09029). The green box indicates the aspartate aminotransferase superfamily (fold type I) domain of pyridoxal phosphate-dependent enzymes (cl18945). The box with zigzag edges indicates the truncated region. Black and red numbers denote distances to the start codon in wild type and mutants, respectively. (B) Alas1 protein was absent in alas $1^{12 / 12}$ mutants. Examination of Alas 1 protein expression in the whole fish body by western blotting at 5 days post fertilization (dpf). GAPDH was used as the loading control. (C) The Sudan black B (SB) signal was absent in alas $1^{12 / 12}$ mutants. SB staining in siblings (upper) and alas $1^{12 / 12}$ mutants (lower) at $3 \mathrm{dpf}$. Boxed regions are magnified in the lower right-hand corner. Scale bars: $200 \mu \mathrm{m}$. (D) SB signal was absent in alas $1^{\text {smu } 350 / \Delta 2}$ mutants. SB staining in siblings (upper) and alas $1^{\text {smu } 350 / \Delta 2}$ mutants (lower) at 3 dpf. Boxed regions are magnified in the lower right-hand corner. Scale bars: $200 \mu \mathrm{m}$. 


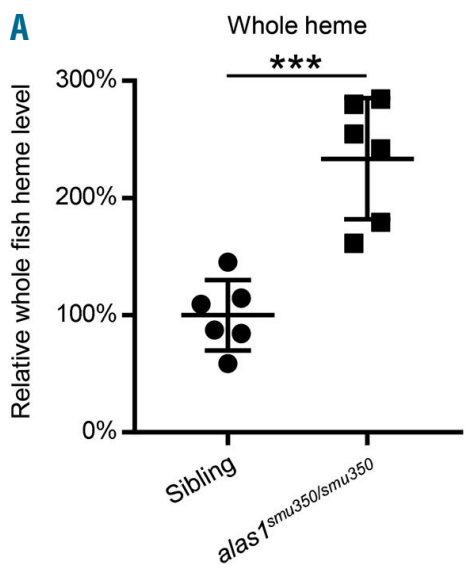

C lyz:DsRed neutrophil heme

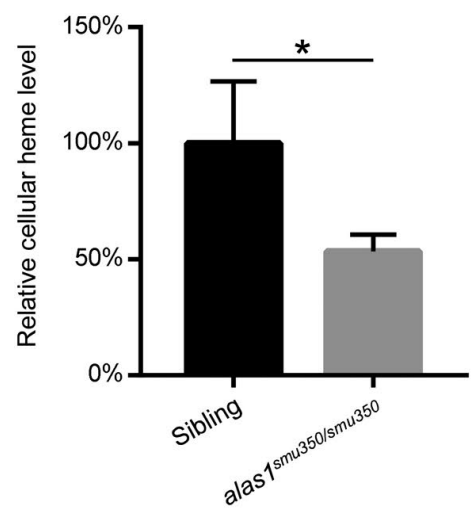

$\mathbf{E}$

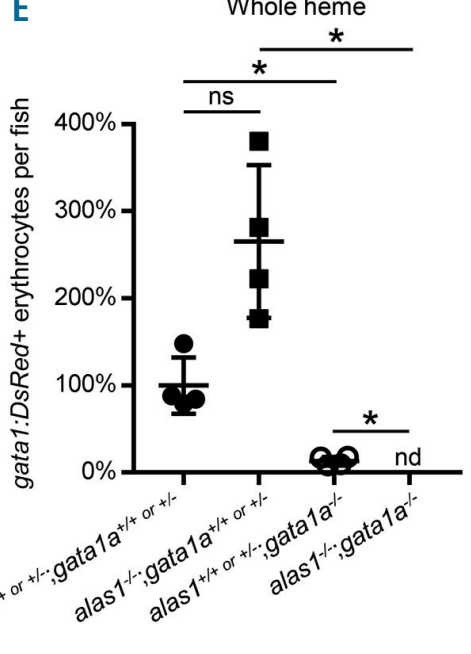

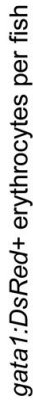

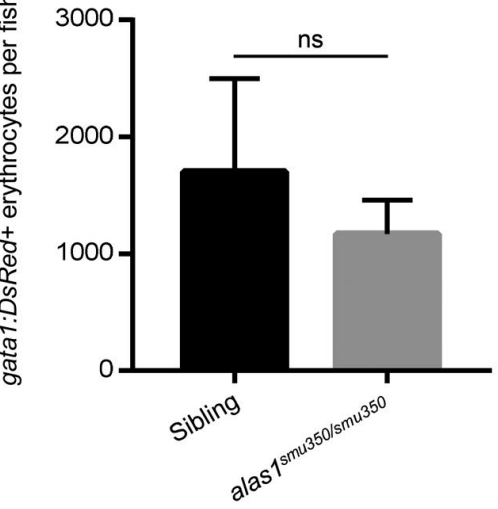

D

gata1:DsRed ${ }^{+}$erythrocyte heme

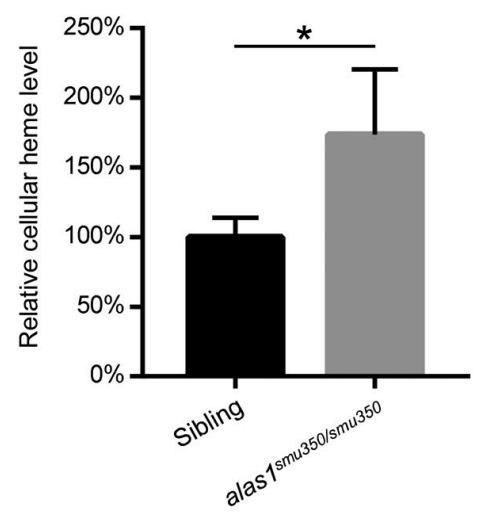

$\mathbf{F}$

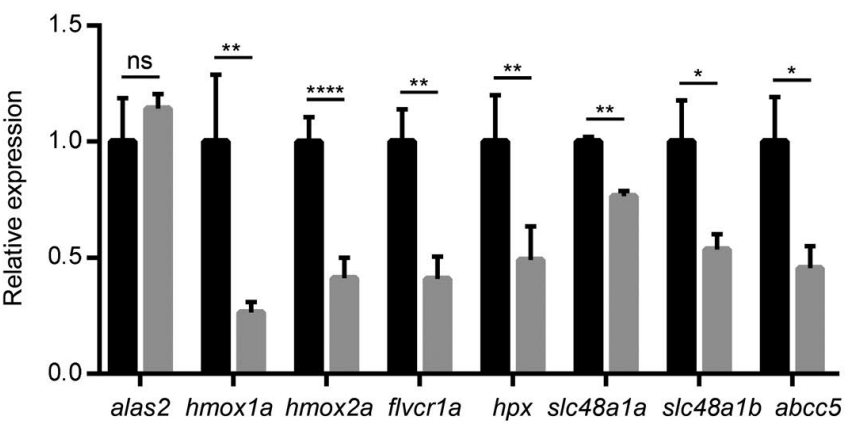

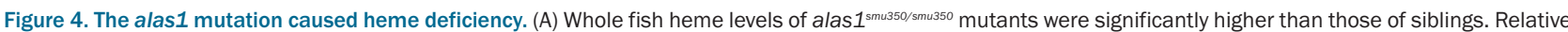
whole fish heme levels in siblings (circles) and alas $1^{\text {smu350/smu350 }}$ mutants (squares) at 4 days post fertilization (dpf). Lines show mean \pm Standard Deviation (SD), 6 individual data points in each group, each data point was based on 3 measurements for 5 embryos; Student $t$-test, $* * * P<0.001$. The relative heme level was normalized to per-fish level. (B) No significant differences in erythrocyte numbers between siblings and alas $1^{\text {smu350/smu350 }}$ mutants. gata1:DsRed ${ }^{+}$erythrocyte numbers were meas-

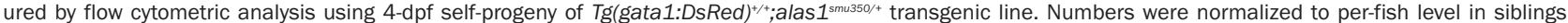
(black column) and alas1 $1^{\text {smu350/smu350 }}$ mutants (gray column). Mean $\pm S D$; performed in triplicate; Student $t$-test, ns: not significant. (C) Cellular heme levels of alas $1^{\text {smu350/smu350 }}$ mutant neutrophils were decreased compared with those of siblings. Relative cellular heme levels in sorted neutrophils (lyz:DsRed ${ }^{+}$cells) of siblings (black column) and alas $1^{\text {smu350/smus5o }}$ mutants (gray column) at $4 \mathrm{dpf}$. Mean \pm SD; performed at least in triplicate; Student $t$-test, $* P<0.05$. The relative heme level was

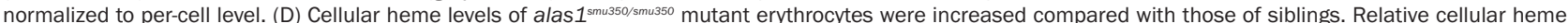

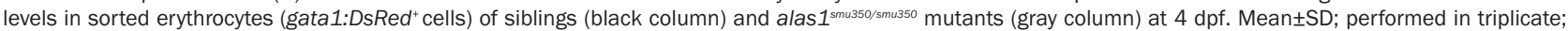

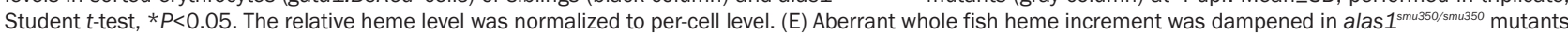
without erythrocytes. Relative whole fish heme levels in 4-dpf alas1;gata1a siblings, alas1 single mutants, gata1a single mutants, and alas1;gata1a double mutants from alas $1^{\text {smu } 350 /+}$; gata $1 a^{m 651 /+}$ in-cross. Lines show mean \pm SD, 4 individual data points in each group, each data point was based on 3 measurements for 5 embryos; one-way ANOVA followed by Dunnett T3 post test, ${ }^{*} P<0.05$. ns: not significant; nd: not detectable. The relative heme level was normalized to per-fish level. (F) Relative expressions of alas 2 and genes related to heme degradation and transport. The assay was performed by RT-qPCR in 4-dpf siblings (black column) and alas $1^{\text {smu } 350 / \text { smu350 }}$ mutants (gray column). Mean $\pm \mathrm{SD} ; \mathrm{n}=8$ in each group, performed in triplicate. Statistical significance was determined using Student $t$-test; $* * * * P<0.0001$, $\star * P<0.01, * P<0.05$. ns: not significant. 
found no significant differences (Figure 4B), indicating the elevated whole heme in mutants was not due to increased erythrocyte numbers. We next isolated neutrophils and erythrocytes of alas $1^{\text {smu } 350 / \text { smu }_{3} 50}$ mutants and their siblings by fluorescence-activated cell sorting (FACS) using 4-dpf selfprogeny of $\operatorname{Tg}(\text { lyz:DsRed })^{+++}$; alas $1^{\text {smusso/+ }}$ and $\operatorname{Tg}$ (gata1:DsRed $)^{+++}$; alas $1^{\text {smul } 350 /+}$ transgenic lines, respectively, to directly measure heme levels in the two cell types. By comparing relative heme levels in neutrophils or erythrocytes between alas $1^{\text {smu } 350 / \text { smu }_{3} 30}$ mutants and their siblings, we found that heme was less abundant in neutrophils of alas $1^{\text {smu } 350 / \text { smu } 350}$ mutants than that of siblings (Figure 4C), while in erythrocytes, heme was more accumulated in erythrocytes of mutants (Figure 4D). These data indicate that alas 1 mutation results in heme insufficiency in neutrophils but abnormal accumulation in erythrocytes. To further confirm that the elevated heme of the whole body was derived from erythrocytes in alas $1^{\text {smu } 350 / \text { smu } 350}$ mutants, we introduced $v 1 t^{m 651,34}$ (a gata1a mutant with a 'bloodless' phenotype having no erythrocytes but intact white blood cells) into the smu350 mutant background to eliminate the effect of erythrocytes. As expected, whole fish heme levels of alas $1^{\text {smu } 350 / \text { smu } 350}$ mutants were almost undetectable compared with those of siblings in the gatala mutant background (Figure 4E). These data indicate that the aberrant heme accumulation of the whole body is indeed derived from erythrocytes in alas $1^{\text {smu } 350 / \text { smu } 350}$ mutants.

Alas2, the other isozyme of Alas1, is essential for the heme biosynthesis in erythrocytes and predominantly expressed in erythrocytes. ${ }^{10}$ To test whether the erythroid heme increment resulted from the elevated alas 2 expres- sion, we checked alas2 expression in alas $1^{\text {smu } 350 / \text { smu } 350}$ mutants. The data showed that alas2 was not altered compared with that in siblings (Figure 4F), suggesting that the erythroid heme accumulation was not due to the compensatory of alas2, at least at the transcription level. Since heme content is tightly controlled by the homeostasis of heme biosynthesis, degradation, and transport pathways, ${ }^{35}$ we then detected the expression of heme oxygenase enzymes (hmox $1 a$ and hmox $2 a),{ }^{10,36}$ which encode the rate-limiting enzymes for heme degradation. The results showed that both gene expressions were down-regulated in alas $1^{\text {smu } 350 / \text { smuзso }}$ mutants (Figure 4F), suggesting the impaired heme degradation in the absence of alas1. The results suggest that the dysregulation of heme biosynthesis affects the heme degradation in alas $1^{\text {smu } 350 / \text { smu } 350}$ mutants, and the elevated heme might be attributed to the reduced heme degradation. The elevated heme in erythrocytes could not be utilized by heme-deficient neutrophils in alas1 mutants, which is likely due to the fact that the synthesized heme in erythrocytes could not be transported to neutrophils. To test this hypothesis, we further detected the expressions of genes encoding heme transporters. Flvcr1a is reported to export heme out of the cell as a plasma membrane heme exporter. ${ }^{37} \mathrm{Hpx}$, a high-affinity heme-binding protein, is reported to interact with FLVCR in heme transfer..$^{35}$ HRG-1 is reported to deliver heme to the cytosol, ${ }^{38}$ which is encoded by slc48a1a (heme transporter hrg1-B) and slc48a1b (heme transporter hrg1-A) in zebrafish. MRP-5/ABCC5 is reported to reside on the plasma membrane and endosomal compartments and regulate the export of cytosolic heme. ${ }^{39}$ RT-qPCR showed that the
A

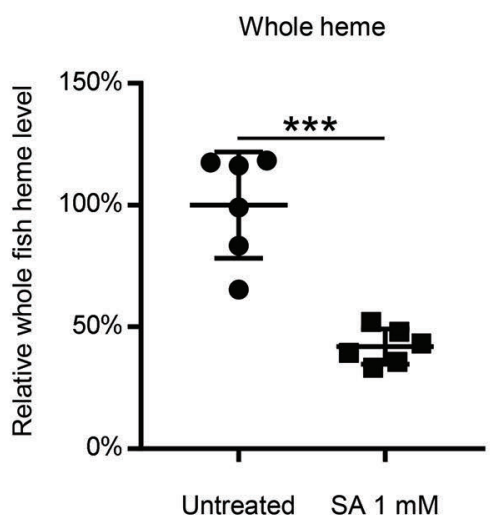

WT 2 dpt Untreated

B

c

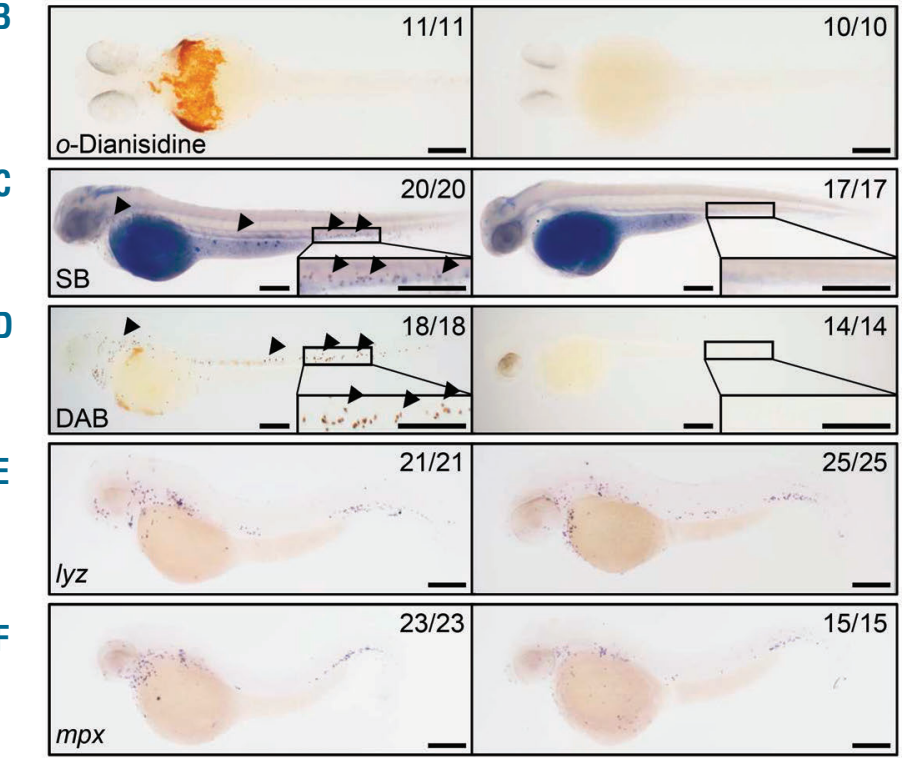

$\mathrm{SA} 1 \mathrm{mM}$

Figure 5. Heme was essential for neutrophil maturation. (A) Whole fish heme levels of succinylacetone (SA)-treated embryos were significantly decreased than those of untreated control. Relative whole fish heme levels in untreated control (circles) and SA-treated embryos (triangles) at 2 days post treatment (dpt). Lines show Mean \pm Standard Deviation (SD), 6 individual data points in each group, each data point was based on 3 measurements for 5 embryos; Student $t$-test: $\star \star \star P<0.001$. The relative heme level was normalized to per-fish level. (B) The o-Dianisidine signal was totally absent in SA-treated embryos. 0 -Dianisidine staining in untreated wild-type (left, 11 of 11 embryos) and SA-treated (right, 10 of 10 embryos) embryos at 2 dpt. (C) The Sudan black B (SB) signal was totally absent in SA-treated embryos. SB staining in untreated wild-type (left, 20 of 20 embryos) and SA-treated (right, 17 of 17 embryos) embryos at 2 dpt. Black arrowheads indicate the neutrophil signals. The boxed regions are magnified in the lower right-hand corner. (D) The DAB signal was totally absent in SA-treated embryos. DAB staining in untreated wild-type (left, 18 of 18 embryos) and SA-treated (right, 14 of 14 embryos) embryos at 2 dpt. Black arrowheads indicate the neutrophil peroxidase signals. The boxed regions are magnified in the lower right-hand corner. (E) WISH of lyz expression in untreated wild-type (left, 21 of 21 embryos) and SA-treated (right, 25 of 25 embryos) embryos at $2 \mathrm{dpt}$. (F) WISH of mpx expression in untreated wild-type (left, 23 of 23 embryos) and SA-treated (right, 15 of 15 embryos) embryos at 2 dpt. Scale bars: $200 \mu \mathrm{m}(\mathrm{B}-\mathrm{F})$. 
A
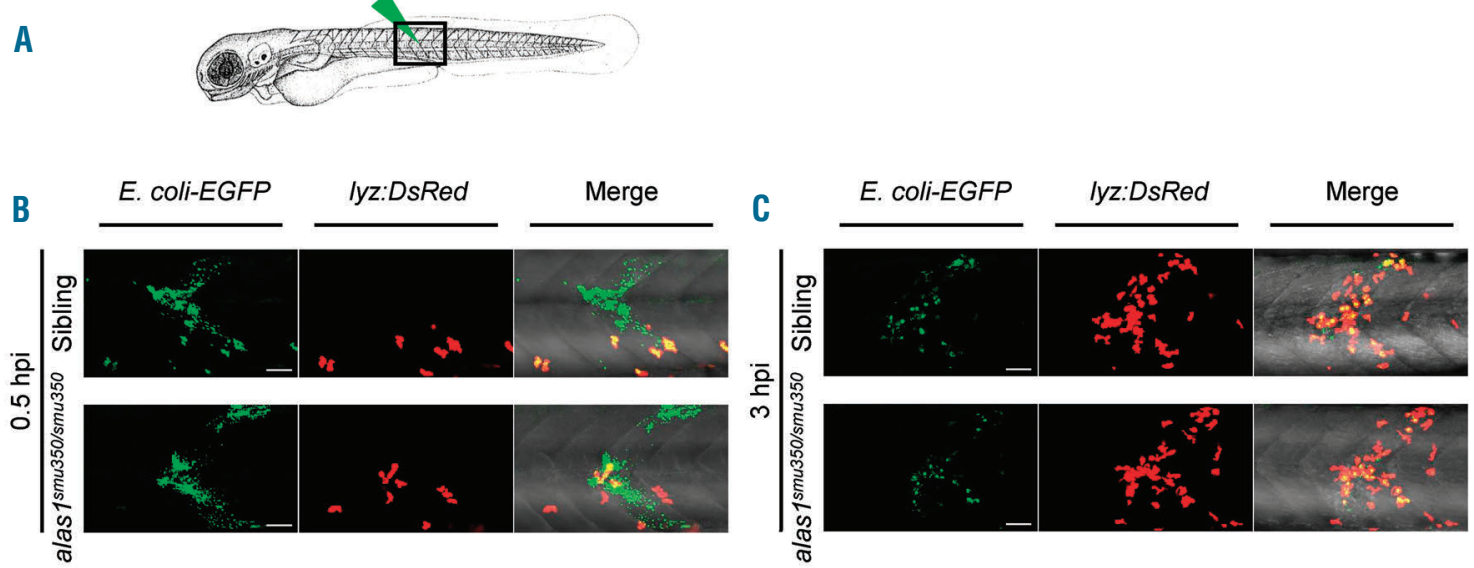

D

$\mathrm{E}$
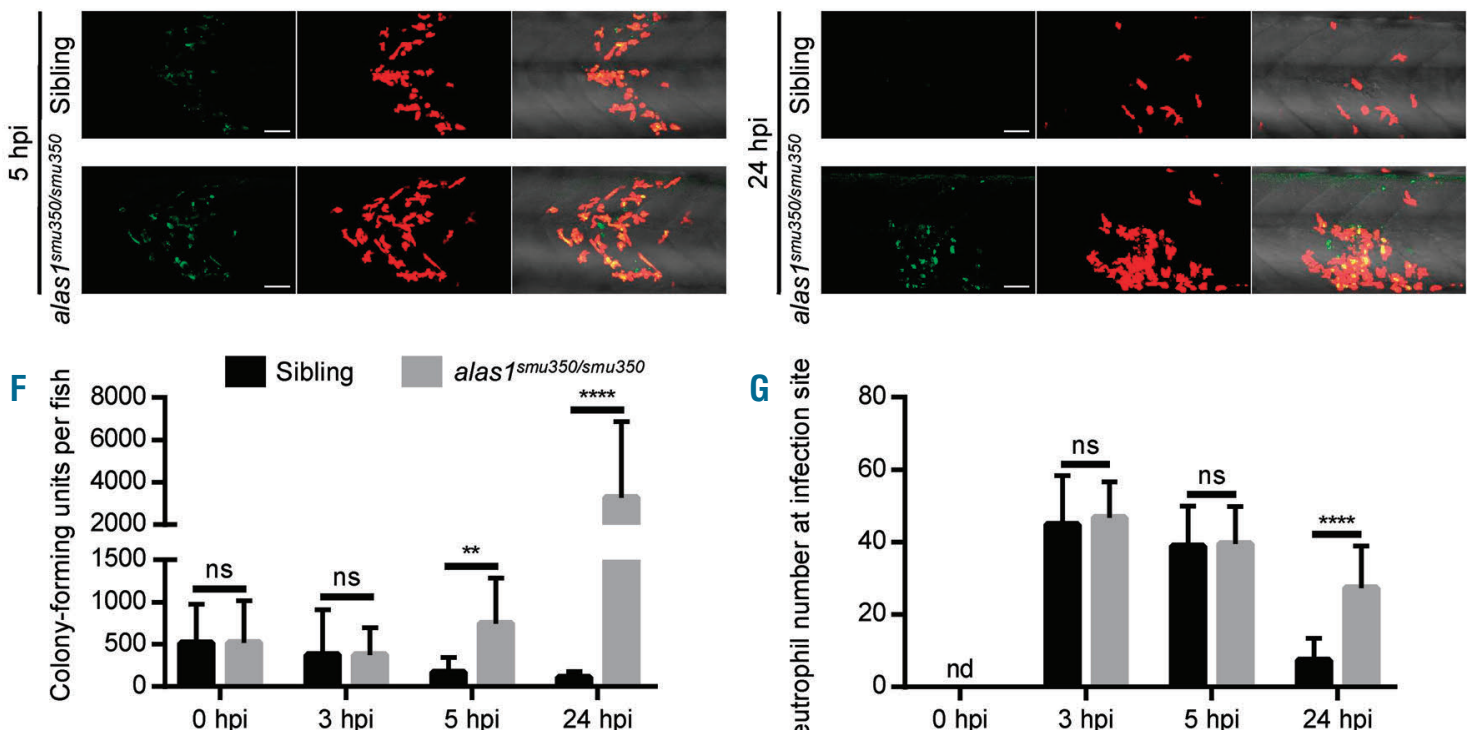

G

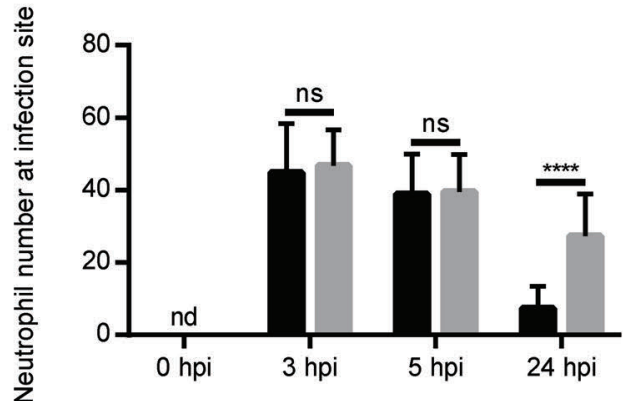

H
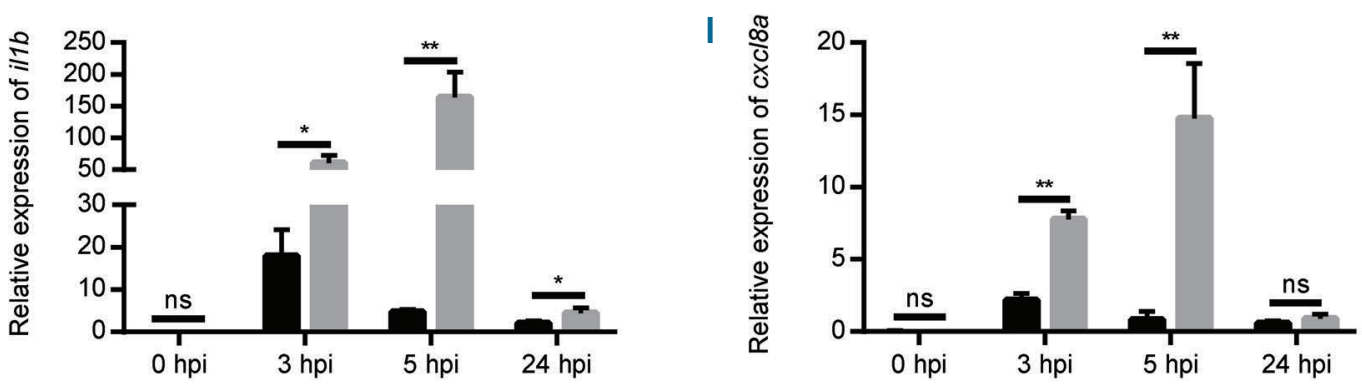

Figure 6. alas1 deficiency caused impaired host immunity against $E$. coli infection. (A) Arrowhead indicates the site of bacteria injection. The imaged region is boxed. (B-E) The in vivo behavior of neutrophils against $E$. coli was monitored by confocal microscopy. eGFP ${ }^{+} E$. coli were subcutaneously injected over one somite into 3-day post fertilization (dpf) sibling (upper panels) and alas $1^{\text {smu350/smu350 }}$ mutant (lower panels) larva of the Tg(lyz:DsRed) background. Neutrophil behavior was analyzed through live imaging at 0.5 hours post injection (hpi) (B), $3 \mathrm{hpi} \mathrm{(C),} 5 \mathrm{hpi}(\mathrm{D})$, and $24 \mathrm{hpi} \mathrm{(E).} \mathrm{All} \mathrm{images} \mathrm{are} \mathrm{maximum-intensity} \mathrm{projections} \mathrm{from} 25$ steps

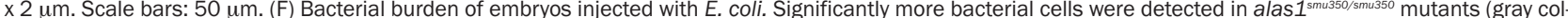
umn) compared with siblings (black column) at 5 and 24 hpi. Mean \pm Standard Deviation (SD), $\mathrm{n}>10$ in each group; Mann-Whitney $U$ test: $* * * * P<0.0001, * * P<0.01$, ns: not significant. (G) Quantification of recruited DsRed ${ }^{+}$neutrophils at infection sites in live embryos injected with $E$. coli. Significantly more neutrophils were observed at the infection sites of alas $1^{\text {smu350/smu } 350}$ mutants (gray column) compared with siblings (black column) at $24 \mathrm{hpi}$. Mean \pm SD; $\mathrm{n}>19$ in each group; Student $t$-test: $* * * * P<0.0001$. nd: not detectable, ns: not significant. $(\mathrm{H})$ Relative $i / 1 b$ expression assessed by RT-qPCR. il1b expression was up-regulated in alas $1^{\text {smu } 350 / \text { smu350 }}$ mutants (gray column) compared with siblings (black column) at 3, 5, and 24 hpi. Mean $\pm S D ; n=4$ in each group, performed in triplicate. Expression levels were adjusted for trauma [phosphate buffered saline (PBS) injection only]. Statistical significance was determined using Student $t$-test: $* * P<0.01$, $* P<0.05$. ns: not significant. (I) Relative cxcl8a expression assessed by RT-qPCR. cxcl8a expression was up-regulated in alas $1^{\text {smu } 350 / \text { smu } 350}$ mutants (gray column) compared with siblings (black column) at 3 and 5 hpi. Mean $\pm S D ; n=4$ in each group, performed in triplicate. Expression levels were adjusted for trauma (PBS injection only). Statistical significance was determined using Student $t$-test: $* * P<0.01$. ns: not significant. 
expressions of these heme transporter genes were decreased in alas $1^{\text {smu } 350 / \text { smu }^{3} 50}$ mutants compared with their siblings (Figure 4F), probably due to the feedback regulation of aberrant heme contents in alas $1^{\text {smu } 350 / \text { smu } 350}$ mutants. Thus, the heme transport deficiency might be one of the reasons that the elevated erythroid heme could not be utilized by neutrophils in alas $1^{\text {smu } 350 / \text { smu } 350}$ mutants.

\section{Heme was essential for neutrophil maturation}

To confirm whether the neutrophil maturation defects were caused by inadequate heme levels, we next treated wild-type zebrafish embryos with SA, an inhibitor of $\delta$-aminolevulinic acid dehydratase, which catalyzes the second step in heme biosynthesis pathway, ${ }^{40}$ to inhibit the endogenous heme levels. Total heme levels of SA-treated embryos were significantly decreased compared with untreated control embryos (Figure 5A). As reported, the $o$-Dianisidine staining signal was decreased as hemoglobin synthesis is inhibited without heme (Figure 5B). ${ }^{23,40}$ When we monitored the neutrophil phenotypes, we found that SA-treated embryos showed loss of $\mathrm{SB}$ and $\mathrm{DAB}$ staining but intact $l y z$ and $m p x$ expression (Figure 5C-F), which mimics the neutrophil maturation defects in Alas1-deficient mutants. These data suggest that the neutrophil defects in alas $1^{\text {smu } 350 / \text { smu } 350}$ mutant are indeed caused by inadequate effective heme in neutrophils.

\section{Neutrophil bactericidal defects in alas $1^{\text {smu } 350 / \text { smu } 350}$ mutants}

Neutrophils play key roles in various functions, including action against certain infections, largely depending on granule proteins. ${ }^{1}$ The neutrophil granule defects suggest that the anti-infection ability of alas $1^{\text {smu } 350 / \text { smu } 350}$ mutant neutrophils may be attenuated. To detect whether alas1 deficiency affected neutrophil bactericidal function, alas $1^{\text {smu } 350 / \text { smu } 350}$ mutants were challenged with a bacterial infection. ${ }^{26,30}$ We subcutaneously injected eGFP labeled $E$. coli over one somite in 3-dpf $\operatorname{Tg}(l y z: D s R e d)$;alas $1^{\text {smu } 350 /+}$ intercrossed embryos, in which DsRed was expressed specifically in neutrophils (Figure 6A). Neutrophil behavior and immune responses were then monitored. We first monitored in vivo bacterial growth and detected the kinetic curves of the bacterial burden of infected embryos. In sibling embryos, bacteria growth was inhibited effectively in the host, as green fluorescent bacteria decreased rapidly in the infection site (Figure 6B-E). By further plating the homogenized embryos/larvae on LB medium for quantification, we found that bacterial colonies were gradually decreased from $5 \mathrm{~h}$ post injection (hpi) and eventually became almost absent at 24 hpi (Figure 6F), suggesting the inhibition of bacterial growth in the host. In mutant embryos, the eGFP bacterial load was similar to siblings within the first 3 hpi (Figure $6 \mathrm{~B}$ and $\mathrm{C}$ ), but fluorescent bacteria were still accumulating at $5 \mathrm{hpi}$ and persisted at 24 hpi, when clearance had been completed in the siblings (Figure 6D-E). Quantification data consistently showed that the plated colony numbers from mutants were similar to those of siblings within the first $3 \mathrm{hpi}$, but the numbers were significantly higher at 5 and 24 hpi than those of the siblings (Figure 6F), suggesting antimicrobial activity was impaired in alas $1^{\text {smu } 350 / \text { smu } 350}$ mutants. We further counted the number of neutrophils recruited to the infection site. Within the first 5 hpi, alas $1^{\text {smu } 350 / \text { smu }_{3} 30}$ embryos showed similar neutrophil recruitment to sibling embryos (Figure 6B-
D, G). However, neutrophils were still accumulating at the infection site in alas $1^{\text {smu } 350 / \text { smu } 350}$ larvae at $24 \mathrm{hpi}$, when recruited neutrophils had almost completely disappeared in the siblings (Figure $6 \mathrm{E}$ and $\mathrm{G}$ ), confirming the defect in neutrophil-specific antibacterial response in alas $1^{\text {smu } 350 / \mathrm{smu} u 30}$ mutants.

To gain further insight into the infection-induced inflammatory alterations, we detected the expression of inflammatory factors $i l 1 b$ and $c x c l 8 a,{ }^{41,42}$ which induce neutrophil chemotaxis and promote immune responses. Expression analyses showed that both genes were significantly up-regulated in infected alas $1^{\text {smu } 350 / \text { smu } 350}$ mutants compared with siblings. The illb and cxclsa expressions peaked in the mutants at $5 \mathrm{hpi}$, at which point their expressions had already been down-regulated in the siblings (Figure 6H and I), suggesting a more dramatic inflammatory response in alas $1^{\text {smu } 350 / \text { smu } 350}$ mutants. Taken together, these data demonstrate that alas1 deficiency causes impaired immune responses to bacterial infection.

\section{Discussion}

In this study, we showed a role for the heme biosynthesis pathway enzyme Alas1 in regulating neutrophil maturation and function. Neutrophils in Alas1-deficient zebrafish had heme deficiency, which led to the loss of heme-related granule protein activities, defective granule formation, and altered immune responses against pathogenic bacteria.

Here, we found that the heme dysregulation caused by the alas1 mutation led to neutrophil defects in zebrafish. Given the important role of neutrophils in immunity, it was expected that Alas1-deficient zebrafish would show impaired bactericidal ability. Inflammatory factors, such as $i l 1 b$ and $c x c l 8 a,{ }^{41,42}$ mediate neutrophil recruitment and promote immune responses. The over-elevated expression of inflammatory factors in infected alas $1^{1 \mathrm{~mm} u 350 / \mathrm{smu}_{3} 30}$ mutants might be explained by the ineffectiveness of killing bacteria, thereby leading to greater pathogen growth and stronger immune responses in the host. We also noticed that the inflammatory responses eventually subsided while the bacterial burden and recruited neutrophils were still present at 24 hpi in Alas1-deficient zebrafish, so it was likely that the inflammatory factors had been excessively depleted.

As far as we know, the regulatory genes and pathways of heme biosynthesis, degradation and transport are largely conserved between mammals and zebrafish in general. ${ }^{20,23,35,36,43}$ Vertebrates contain two ALAS isozymes encoded by 2 distinct genes located on different chromosomes; ALAS2 is expressed in erythrocytes, whereas ALAS1 is ubiquitously expressed. ${ }^{11}$ By searching the integrated RNA-seq database on BloodSpot, ${ }^{44}$ we found that, in humans and mice, ALAS1 is highly expressed in myeloid cells, which is consistent with our zebrafish data and partly explains the importance of alas1 for neutrophils. In mice, ALAS1 is also highly expressed in the liver, exocrine, and endocrine glands, suggesting specific roles in those tissues. ${ }^{24}$ Accordingly, even though alas1 is ubiquitously expressed, we suspect that alas 1 may play specific roles in certain tissues to meet the need of hemoproteins. It is reported that $A L A S 1$-null mice died in utero until E8.5, with a severely retarded morphology, indicating that ALAS1 is essential for the early development of mouse embryos. ${ }^{24}$ 
It is likely that because of the lethality resulting from ALAS1 deficiency, no reported human diseases directly caused by mutations in ALAS1 have been reported so far. Although the Alas1-deficient zebrafish were indistinguishable on morphology from wild type at embryonic stages, the Alas1-deficient zebrafish are not viable past 8 $\mathrm{dpf}$ and showed some morphological defects from $4 \mathrm{dpf}$ onwards, such as delayed disappearance of the yolk sac, abnormal liver, and failed swim bladder formation (data not shown). Thus, the specific functions of ALAS1 in different tissues and organs remain to be clarified.

Alas1-deficient zebrafish showed impaired heme levels in neutrophils but elevated heme levels in erythrocytes. It is known that in addition to mitochondrial heme synthesis within all cells, heme can also be transported in and out of cells from the plasma. ${ }^{11}$ Intracellular heme levels are tightly controlled through the co-ordination of heme synthesis, degradation, and trafficking. ${ }^{35}$ In this study, we found that the expression of alas2, encoding the other rate-limiting enzyme of heme synthesis, was not changed, while the expressions of heme degradation and transporter genes were down-regulated in alas $1^{\text {smmu } 350 / \mathrm{smu}_{3} 50}$ mutants. In Alas1-deficient zebrafish, cellular heme levels were unexpectedly elevated in erythrocytes, partly due to the decrease in heme degradation. The excessive heme could not be effectively used by neutrophils, probably because the heme transport was impaired. Similar to mice, ${ }^{24}$ the over-produced heme by alas 2 in erythrocytes could not compensate for the function of alas 1 in zebrafish, indicating the essential roles of alas1. The feedback mechanisms for heme homeostasis remain unclear, so future studies will be needed to elucidate the molecular mechanisms of heme metabolisms and trafficking.

Impaired heme biosynthesis or heme deficiency leads to heme-related disorders, such as anemias, acute porphyrias, and leukemia. ${ }^{11}$ Acute intermittent porphyria is characterized by the accumulation and/or excretion of excess heme precursors. ${ }^{11}$ As Alas1 is the key enzyme in heme biosynthesis, repressing Alas1 activity by RNAi is now being used to prevent acute porphyria attacks. 45,46 Thus, Alas1-deficient zebrafish may serve as an in vivo animal model for evaluating the risks of therapeutic strategies, since Alas1 deficiency causes neutrophil defects, as well as other potential defects in Alas1-abundant tissues. This study may also contribute to the development of new drugs or treatment strategies for hemerelated diseases.

\section{Acknowledgments}

We thank Dr. Zilong Wen for sharing the Tg(gata1:DsRed) line and eGFP-labeled E. coli strain XL10.

\section{Funding}

This work was supported by the National Natural Science Foundation of China (31471378) and the Team Program of the Guangdong Natural Foundation (2014A030312002).

\section{References}

1. Amulic B, Cazalet C, Hayes GL, Metzler KD, Zychlinsky A. Neutrophil function: from mechanisms to disease. Annu Rev Immunol. 2012;30(1):459-489.

2. Odobasic D, Kitching AR, Holdsworth SR. Neutrophil-mediated regulation of innate and adaptive immunity: The role of myeloperoxidase. J Immunol Res. 2016; 2016(6):2349817

3. Cowland JB, Borregaard N. Granulopoiesis and granules of human neutrophils. Immunol Rev. 2016;273(1):11-28.

4. Levy $\bigcirc$. Antimicrobial proteins and peptides: anti-infective molecules of mammalian leukocytes. J Leukoc Biol. 2004; 76(5):909-925

5. Bennett CM, Kanki JP, Rhodes J, et al. Myelopoiesis in the zebrafish, Danio rerio. Blood. 2001;98(3):643-651.

6. Xu J, Du L, Wen Z. Myelopoiesis during zebrafish early development. J Genet Genomics. 2012;39(9):435-442.

7. Jin H, Huang Z, Chi Y, et al. c-Myb acts in parallel and cooperatively with Cebp1 to regulate neutrophil maturation in zebrafish. Blood. 2016;128(3):415-426.

8. Wang $\mathrm{K}$, Fang $\mathrm{X}, \mathrm{Ma} \mathrm{N}$, et al. Myeloperoxidase-deficient zebrafish show an augmented inflammatory response to challenge with Candida albicans. Fish Shellfish Immunol. 2015;44(1):109-116.

9. Di $Q$, Lin $Q$, Huang Z, et al. Zebrafish nephrosin helps host defence against Escherichia coli infection. Open Biol. 2017; 7(8).

10. Furuyama K, Kaneko K, Vargas PD. Heme as a magnificent molecule with multiple mis- sions: Heme determines its own fate and governs cellular homeostasis. Tohoku J Exp Med. 2007;213(1):1-16.

11. Tsiftsoglou AS, Tsamadou AI, Papadopoulou LC. Heme as key regulator of major mammalian cellular functions: Molecular, cellular, and pharmacological aspects. Pharmacol Ther. 2006;111(2):327345.

12. Yamamoto M, Hayashi N, Kikuchi G. Evidence for the transcriptional inhibition by heme of the synthesis of delta-aminolevulinate synthase in rat liver. Biochem Biophys Res Commun. 1982;105(3):985990.

13. Suzuki H, Tashiro S, Hira S, et al. Heme regulates gene expression by triggering Crm1dependent nuclear export of Bach1. EMBO J. 2004;23(13):2544-2553.

14. Tahara T, Sun JY, Nakanishi K, et al. Heme positively regulates the expression of betaglobin at the locus control region via the transcriptional factor Bach1 in erythroid cells. J Biol Chem. 2004;279(7):5480-5487.

15. Yamamoto M, Hayashi N, Kikuchi G. Translational inhibition by heme of the synthesis of hepatic delta-aminolevulinate synthase in a cell-free system. Biochem Biophys Res Commun. 1983;115(1):225-231.

16. Chen JJ, London IM. Regulation of protein synthesis by heme-regulated eIF-2 alpha kinase. Trends Biochem Sci. 1995;20(3):105108.

17. Ishikawa $\mathrm{H}$, Kato $\mathrm{M}$, Hori $\mathrm{H}$, et al. Involvement of heme regulatory motif in heme-mediated ubiquitination and degradation of IRP2. Mol Cell. 2005;19(2):171-181.

18. Kubota Y, Nomura K, Katoh Y, Yamashita R Kaneko K, Furuyama K. Novel mechanisms for heme-dependent degradation of ALAS1 protein as a component of negative feedback regulation of heme biosynthesis. J Biol Chem. 2016;291(39):20516-20529.

19. Faller M, Matsunaga M, Yin S, Loo JA, Guo F. Heme is involved in microRNA processing. Nat Struct Mol Biol. 2007;14(1):23-29.

20. Ferreira GC. Heme biosynthesis: biochemistry, molecular biology, and relationship to disease. J Bioenerg Biomembr. 1995;27(2):147-150.

21. May A, Bishop DF. The molecular biology and pyridoxine responsiveness of X-linked sideroblastic anaemia. Haematologica. 1998;83(1):56-70.

22. Yamamoto M, Nakajima O. Animal models for X-linked sideroblastic anemia. Int J Hematol. 2000;72(2):157-164.

23. Brownlie A, Donovan A, Pratt SJ, et al. Positional cloning of the zebrafish sauternes gene: a model for congenital sideroblastic anaemia. Nat Genet. 1998; 20(3):244-250.

24. Okano S, Zhou L, Kusaka $\mathrm{T}$, et al. Indispensable function for embryogenesis, expression and regulation of the nonspecific form of the 5-aminolevulinate synthase gene in mouse. Genes Cells. 2010;15(1):7789.

25. Henry KM Loynes CA Whyte MKB Renshaw SA. Zebrafish as a model for the study of neutrophil biology. J Leukoc Biol. 2013;94(4):633-642

26. Colucci-Guyon E, Tinevez JY, Renshaw SA Herbomel P. Strategies of professional phagocytes in vivo: unlike macrophages, neutrophils engulf only surface-associated microbes. J Cell Sci. 2011;124(18):30533059.

27. Wang K, Huang Z, Zhao L, et al. Large-scale 
Regulation of neutrophil maturation in zebrafish

forward genetic screening analysis of development of hematopoiesis in zebrafish. J Genet Genomics. 2012;39(9):473-480.

28. Westerfield M. The zebrafish book. A guide for the laboratory use of zebrafish (Danio rerio). th ed. Univ. of Oregon Press, Eugene, 2000.

29. Hall C, Flores MV, Storm T, Crosier K, Crosier P. The zebrafish lysozyme C promater drives myeloid-specific expression in transgenic fish. BMC Nev Biol. 2007;7(1):42.

30. Benard EL, van der Soar AM, Ellett F, Lieschke GJ, Spain HP, Meijer AH. Infection of zebrafish embryos with intracellular batteral pathogens. J Vis Exp. 2012;15(61):3781.

31. Le Guyader D, Redd MJ, Colucci-Guyon E, et al. Origins and unconventional behavior of neutrophils in developing zebrafish. Blood. 2008;111(1):132-141.

32. Wang SF, Xu XP, Lin ZH, et al. Hemoglobin likely function as peroxidase in blood clam Tegillarca granosa hemocytes. J Immunol Res. 2017;2017(8):7125084.

33. Sadlon TJ, Dell'Oso T, Surinya KH, May BK. Regulation of erythroid 5-aminolevulinate synthase expression during erythropoiesis. Int J Biochem Cell Biol. 1999;31(10):11531167.

34. Lyons SE, Lawson ND, Lei L, Bennett PE, Weinstein BM, Li PP. A nonsense mutation in zebrafish gata1 causes the bloodless the- neotype in vlad topes. Proc Natl Arad Sci USA. 2002;99(8):5454-5459.

35. Khan AA, Quigley JG. Control of intracellular heme levels: heme transporters and heme oxygenates. Biochim Biophys Alta. 2011;1813(5):668-682.

36. Holowiecki A, O'Shields B, Jenny MJ. Spatiotemporal expression and transcriptional regulation of heme oxygenase and biliverdin reductase genes in zebrafish (Danio rerio) suggest novel roles during early developmental periods of heightened oxidative stress. Comp. Biochem. Physiol. C Toxicol. Pharmacol. 2017;191:138-151.

37. Mercuric S, Petrillo S, Chiabrando D, Bass ZI, Gays D, Camporeale A, et al. The heme exporter Flvcr1 regulates expansion and ifferentiation of committed erythroid proventors by controlling intracellular heme ecumulation. Haematological. 2015; 100 (6):720729.

38. Rajagopal A, Roo AU, Amigo J, Than M, Upadhyay SK, Hall C, et al. Haem homeostasis is regulated by the conserved and concerted functions of HRG-1 proteins. Nature. 2008;453(7198):1127-1131.

39. Korolnek T, Zhang J, Beardsley S, Scheffer GL, Hamza I. Control of metazoan heme homeostasis by a conserved multidrug resistance protein. Cell Metal. 2014; 19(6):1008-1019.
40. Beru N, Sahr K, Goldwasser E. Inhibition of heme synthesis in bone marrow cells by succinylacetone: effect on globin synthesis. J Cell Biochem. 1983;21(2):93-105.

41. Dinarello CA. Interleukin-1 in the pathogenisis and treatment of inflammatory diseases. Blood. 2011;117(14):3720-3732.

42. de Oliveira S, Reyes-Aldasoro CC, Cancel S, Renshaw SA, Mulero V, Calado A. Cxcl8 (IL8) mediates neutrophil recruitment and behavior in the zebrafish inflammatory response. J Immunol. 2013;190(8):43494359.

43. Ca C, Fleming MD. The ins and outs of erythroid heme transport. Haematological. 2015;100(6):703.

44. Bagger FO, Sasivarevic D, Sohi SH, et al. BloodSport: a database of gene expression profiles and transcriptional programs for healthy and malignant haematopoiesis. Nucleic Acids Res. 2016;44(D1):D917-924.

45. Chan A, Liebow A, Yasuda M, et al. Preclinical development of a subcutaneous ALAS1 RNA therapeutic for treatment of hepatic porphyrias using circulating RNA quantification. Mol Then Nucleic Acids. 2015;4(11):e263.

46. Balwani M, Wang B, Anderson KE, et al. Acute hepatic porphyrias: recommendations for evaluation and long-term management. Hepatology. 2017; 66(4):1314-1322.

haematological | 2018; 103(11)

1795 